\title{
Construindo o Internato de Saúde Mental: a Experiência da Universidade São Francisco
}

\section{Creating a Mental Health Internship Program: the Experience of Universidade São Francisco}

\author{
Luiz Fernando Paulin ${ }^{1}$ (i) \\ Regina Caeli Guerra Poças ${ }^{1}$ (ID \\ Fabio Giraldez $z^{1}$ (I) \\ Juliana Marim ${ }^{\mathrm{I}}$ (D) \\ Ivan Centelhas ${ }^{\mathrm{I}}$ (D) \\ Camila Nicolucci ${ }^{\mathrm{I}}$ i]
}

\section{PALAVRAS-CHAVE}

- Psiquiatria/Educação.

- Currículo.

- Educação Médica.

- Internato de Medicina

- Saúde Mental.

\section{RESUMO}

Introdução: O modelo de formação médica no Brasil vem apresentando importantes modificações nos últimos anos. Com o lançamento do Programa Mais Médicos em 2013, cujo propósito era a formação de recursos humanos na área médica para atuarem preferencialmente em áreas carentes, sob a referência do Sistema Único de Saúde (SUS), e o posterior lançamento das Diretrizes Curriculares Nacionais, no ano seguinte, uma nova realidade se apresentou em relação ao internato médico, quanto à necessidade do módulo de saúde mental. O surgimento de novas escolas médicas, bem como a inexistência de internato de saúde mental em escolas mais antigas, pressupõe a reflexão e viabilização desse novo campo de atuação. O artigo propõe apresentar a experiência do curso de Medicina da Universidade São Francisco (USF), de Bragança Paulista, que desenvolve internato de saúde mental há 15 anos. Método: Como premissa estrutural estabelecemos cinco norteadores de organização, planejamento e execução do internato, respeitando a especificidade e realidade de cada curso no que tange à carga horária, ao corpo docente e aos cenários de aprendizagem. Resultados: O objetivo é apresentar informações sobre o conteúdo programático de saúde mental na graduação, a estruturação do internato, a existência de internato próprio de saúde mental ou vinculado a um módulo mais abrangente, a carga horária existente, a distribuição do número de alunos por módulo, o número de docentes e os cenários de aprendizagem existentes. Conclusão: Os autores apresentam o modelo de funcionamento do internato de saúde mental do curso de Medicina da USF, que deve servir como proposta de viabilização e norteamento para os cursos de Medicina que necessitam de um referencial de formação. A educação médica que se encontra em importante momento de inflexão deve formar profissionais que, mesmo não sendo psiquiatras, sintam-se preparados para as demandas de saúde mental, que se apresentam no cotidiano da atenção à saúde, sob a estruturação dos princípios básicos do SUS. 


\section{KEYWORDS}

- Education/Psychiatry.

- Curriculum

- Medical Education.

- Internship

- Mental Health

\section{ABSTRACT}

Introduction: The medical education model in Brazil has been going through major changes in recent years. With the introduction of the "More Doctors" Program in 2013, which proposes to train human resources in the medical field to work preferentially in Brazil's neediest regions under the reference of the Brazilian Unified Health System (SUS), and the subsequent launching in the following year of the National Curriculum Guidelines for medical courses, a new reality was introduced regarding medical internship and the need for a mental health module. The emergence of new medical schools, as well as the lack of mental health internship programs in older schools, presupposes the necessity of reflection and viability of this new field of activity. This article aims at presenting the experience of the São Francisco University (USF) medical course in Bragança Paulista, which has been developing its mental health internship program for fifteen years. Method: As the structural premise, we established five guiding principles for the organization, planning and implementation of the internship program, respecting the specificity and reality of each course regarding the workload, faculty and learning scenarios. Results: It seeks to know about the programmatic content of mental health in medical courses, the internship structure, the existence of a mental health internship program of its own or related to a more comprehensive module, the existing workload, the number of students distributed among the modules, the number of faculty members and the learning scenarios. Conclusion: The authors present the operating model of the mental health internship program of the medical course at USF, in order to serve as a proposal of viability and guidance for medical courses that seek for a training referential. It is concluded that medical education, which faces an important moment of change-over, can train professionals who, even if they do not become psychiatrists, feel prepared for the mental health demands that can emerge during the healthcare routine and under the basic principles of SUS.

Recebido em 16/8/19

Aceito em 25/9/19

\section{INTRODUÇÃO}

A formação médica no Brasil tem passado por um período de grandes mudanças nos últimos anos, mais especificamente após o lançamento do Programa Mais Médicos, ocorrido em 2013, transformado na Lei n. 12.871 ${ }^{1}$. Tal programa surgiu no intuito de formar recursos humanos na área médica para o Sistema Único de Saúde (SUS), tendo como objetivo maior diminuir a carência desses profissionais em regiões inóspitas, bem como fortalecer a prestação de serviços na atenção básica em saúde no país ${ }^{1,2}$.

A partir das recomendações do Programa Mais Médicos, houve a necessidade de readequação da formação médica no Brasil, o que resultou na elaboração das novas Diretrizes Curriculares Nacionais (DCN) para o curso de graduação em Medicina, promulgadas pela Resolução do Conselho Nacional de Educação e da Câmara de Educação Superior (CNE/CES), de 20 de junho de $2014^{3}$.

As DCN propõem que o graduando de Medicina tenha formação geral, humanística, crítica, reflexiva e ética, com capacidade de atuar nos diferentes níveis de atenção à saúde, com ações de promoção, prevenção, recuperação e reabilitação à saúde, nos âmbitos individual e coletivo, com responsabilidade social e compromisso com a defesa da cidadania, da dignidade humana, da saúde integral do ser humano e tendo como transversalidade em sua prática, sempre, a determinação social do processo saúde-doença ${ }^{3}$.

Se a formação médica do final do século XX era firmada na estrutura hospitalar e ambulatorial, observa-se, a partir do século XXI, um processo gradativo de mudança de cenários de aprendizagem, e as DCN enfatizam a necessidade de a formação ocorrer prioritariamente, mas não exclusivamente, na atenção básica de saúde e nos serviços de urgência e emergência.

Tais práticas promoveram uma mudança no perfil do internato médico e, desde as DCN de $2001^{4}$, vêm apontando para uma nova tendência relacionada ao último estágio na formação médica. As DCN de 2001 propunham que o internato tivesse carga horária mínima equivalente a 35\% da carga horária total da graduação e que fosse centrado nas áreas de clínica médica, cirurgia, ginecologia-obstetrícia, pediatria e saúde coletiva, com atividades eminentemente práticas, com carga horária teórica não superior a $20 \%$ do total do estágio.

As DCN de 2014 acrescentaram novas exigências em relação ao internato médico, como a obrigatoriedade de $30 \%$ da carga horária prevista para o internato médico ocorrer na atenção básica e em serviços de urgência e emergência do SUS. Os 70\% restantes da carga horária deverão ocorrer nas áreas anteriormente estabelecidas pelas DCN de 2001, com o acréscimo da área de saúde mental ${ }^{3,4}$.

De acordo com Cândido e Batista ${ }^{5}$, que analisaram nove escolas médicas do estado do Rio de Janeiro, todos os coordenadores de cursos de Medicina que responderam ao questionário proposto concordaram com a inclusão obrigatória da área de saúde mental, no entanto, até o momento da coleta dos dados de pesquisa (2016), 46\% das escolas médicas pesquisadas não possuíam qualquer atividade de saúde mental no internato.

O objetivo deste trabalho é apresentar a experiência do curso de Medicina da Universidade São Francisco (USF), câmpus de Bragança Paulista, com mais de 15 anos de internato de saúde mental, no intuito de contribuir para que as escolas médicas que tenham a necessidade de estruturar tal programa possam ter algumas referências na formatação dele.

Nossa proposta é desenvolver cinco elementos norteadores para a estruturação da proposta, os quais são apresentados a seguir. 
Existem atividades de ensino de saúde mental na graduação? Elas contemplam conteúdos teóricos e práticos?

Atualmente vivemos uma mudança de paradigma na estrutura curricular dos cursos de Medicina. As DCN de 2014 reiteram a ênfase ampliada de currículo como prática social situada e historicamente condicionada, tendo o SUS como ordenador da formação médica ${ }^{3}$.

É importante frisar a necessidade de temas vinculados à saúde mental na área de competência da atenção à saúde, visto que quadros prevalentes como transtornos depressivos, ansiosos, dependência química e alcoolismo exigem conhecimento e habilidade daquele que se propõe a ser médico.

Não há um momento específico para o desenvolvimento de temas relacionados à saúde mental, porém, em nossa instituição, desde o primeiro semestre do curso, iniciamos abordagens que inserem questões relacionadas à saúde mental do estudante de medicina e do médico em uma disciplina denominada "Introdução ao aprendizado médico".

Outros momentos são desenvolvidos para abordagem da psicologia médica, semiologia psiquiátrica e clínica psiquiátrica, podendo haver ou não disciplina específica de psiquiatria ou saúde mental na graduação. $\mathrm{O}$ curso de Medicina da Universidade Estadual de Campinas (Unicamp), por exemplo, tem, em seu currículo atual, seis disciplinas a cargo do Departamento de Psicologia Médica e Psiquiatria, em que os conteúdos de psiquiatria e saúde mental são desenvolvidos durante o curso ${ }^{6}$.

A maioria das instituições de ensino, no entanto, tem carga horária muito restrita vinculada à saúde mental, porém o importante é conhecer o que é desenvolvido durante a graduação, se as atividades são predominantemente teóricas ou se há vivência prática e qual é o papel do módulo de saúde mental na formação médica.

Deve-se salientar que o internato pressupõe o máximo de $20 \%$ da carga horária com atividades teóricas, algo em torno de oito horas semanais para uma carga horária total de 40 horas semanais, e se faz necessário resgatar temas mais prevalentes para a formação do médico geral, no entanto o internato exige outra concepção pedagógica, em que o exercício do atendimento, a discussão dos casos e a supervisão são fatores intrínsecos a um bom aprendizado. Entendemos que no internato as atividades teóricas possam ocorrer, mas preferencialmente vinculadas às atividades práticas.

O internato está estruturado de que forma? Há internato próprio de saúde mental?

O internato tem se tornado o elo fundamental na formação médica, quando o aluno consegue exercer de fato o treinamento em serviço. É certo que um dos desafios que o ensino médico enfrenta é conseguir integrar $a$ área básica com a clínica em um processo contínuo na formação médica, o que, em geral, não ocorre. Observa-se que a graduação e o internato seriam como dois mundos distintos e dissociados, inclusive em suas práticas pedagógicas, inserção docente e processos avaliativos.

Segundo Marcondes e Mascaretti ${ }^{7}$, há três tipos distintos de internato: rotativo, eletivo e integrado. O internato rotativo é o mais comum, em que o aluno rodizia nas grandes áreas, com carga horária definida previamente e forte influência das especialidades. Estudo de Zanolli, Maciel, Streit e Muraguchi ${ }^{8}$ demonstrou que $94 \%$ das escolas médicas do país possuem esse modelo de internato.

No internato eletivo, o estudante tem autonomia para escolher as áreas de interesse. A questão limitante seria a especialização precoce. No
Brasil, a grande maioria das escolas médicas que possui internato rotativo permite o estágio eletivo, fato que enriquece a formação médica.

Por fim, há o internato integrado, cuja estruturação está relacionada ao cenário de prática e aos modelos de atenção à saúde, como unidade básica de saúde (UBS), maternidade, ambulatório, hospital etc. Tal modelo pode ser incorporado ao internato rotativo quando se define, por exemplo, que no quinto ano o aluno passará por módulos em UBS e ambulatórios e no sexto exercerá as atividades em hospitais.

Observa-se que as DCN de 2014 apresentam uma mescla entre os três tipos de internato, sendo rotativo (ao exigir $70 \%$ da carga nas áreas essenciais), eletivo (ao permitir que $25 \%$ da carga horária total estabelecida possa ocorrer em instituições outras, preferencialmente nos serviços do SUS e que possuam programas de residência médica credenciados pela Comissão Nacional de Residência Médica) e integrado(ao propor que $30 \%$ da carga horária prevista seja desenvolvida na atenção básica e em serviços de urgência e emergência do SUS ${ }^{3}$.

Especificamente em relação à saúde mental, como ainda se prioriza o modelo rotativo, deve-se buscar um módulo próprio, com carga horária equivalente aos outros módulos. É certo que em muitas instituições não há tradição relacionada à saúde mental, e em várias situações, para privilegiar as grandes áreas, a "saúde mental" se torna um tópico de uma área maior, mais frequentemente a clínica médica ${ }^{9}$.

Entendemos que o modelo ideal e de melhor formatação seria o módulo próprio. Se tal situação não for possível, devem-se ampliar as áreas de atuação da saúde mental. Por exemplo, no módulo de pediatria, deve haver a inserção da saúde mental infantil e da adolescência; na ginecologia-obstetrícia, a estruturação da saúde mental da mulher; e na urgência e emergência, treinamento em urgências psiquiátricas.

É certo que pode haver uma mescla de modelos, e em algumas instituições, mesmo havendo internato próprio de saúde mental, entendese que a saúde mental infantil deve estar vinculada à pediatria, e não à saúde mental, por meio de um modelo integrado.

Qual é a carga horária do internato? Qual é o número de alunos no módulo?

As DCN de $2014^{3,5}$, em seu artigo 24 , explicitam com clareza a questão da carga horária do internato, definindo em $35 \%$ da carga horária total do curso. O mínimo de $30 \%$ deve ocorrer na atenção básica e em serviços de urgência e emergência, e os 70\% restantes nas áreas de clínica médica, cirurgia, ginecologia-obstetrícia, pediatria, saúde coletiva e saúde mental.

Vale destacar aqui essa definição, por vezes, é incongruente com a realidade, pois, quando se define a carga horária, trabalha-se com parâmetros distintos, em que atenção básica e urgência e emergência são cenários de atuação, enquanto os $70 \%$ restantes estão divididos em áreas de atuação, fato que gera distorção de entendimento. Poderíamos, por exemplo, definir que, nas áreas de atuação, ao menos $30 \%$ estivessem vinculados aos cenários de aprendizagem da atenção básica e urgência $e$ emergência, o que ficaria mais adequado.

No curso de medicina da USF, a carga horária do internato é de 3.849 horas, a qual é dividida em três módulos por semestre, com carga horária de 320 horas/módulo, em um programa de quatro semestres, a saber ${ }^{10}$ :

- Nono semestre: saúde da criança, saúde da mulher e saúde coletiva (cenários prioritários em UBSs).

- Décimo semestre: saúde do adulto I (clínica médica), saúde do

REVISTA BRASILEIRA DE EDUCAÇ̃̃o MÉDICA

3 $44(1)$ : e005; 2020 
adulto II (clínica cirúrgica) e saúde mental (cenários prioritários em ambulatórios).

- $11^{\circ}$ semestre: pediatria, ginecologia-obstetrícia e estágio eletivo (cenários prioritários hospitalar e unidade de pronto atendimento - UPA).

- $12^{\circ}$ semestre: clínica médica, clínica cirúrgica e urgência/ emergência (cenários prioritários hospitalar, UPA e prontosocorro).

Com uma carga horária definida em 40 horas/semana no internato, o módulo corresponde a cerca de oito semanas, quantidade significativa para o aprendizado em saúde mental. É certo que, como relatamos anteriormente, algumas instituições possuem internato de saúde mental inserido em áreas maiores como clínica médica, porém, a partir das DCN de 2014, o mais adequado seria ter um módulo próprio de saúde mental.

O número de alunos que passam no módulo depende evidentemente do número de ingressantes. Como atualmente em nossa instituição o número de ingressantes é de 44 alunos por semestre, o módulo possui cerca de 15 alunos. Porém, esse número pode ser ampliado, pois não estão computados alunos vinculados a programas de inclusão, como o Programa Universidade para Todos (ProUni). Com a inclusão desses alunos, o número pode aumentar para 16 ou 17 por módulo.

A questão relacionada ao número de alunos por módulo pode parecer ser secundária, porém é fundamental para discutirmos o item a seguir, que está relacionado aos cenários de aprendizagem.

\section{Quais são os cenários de aprendizagem?}

Certamente o ponto central para que o módulo de saúde mental se torne efetivo refere aos cenários de aprendizagem inseridos preferencialmente, se não de forma exclusiva, naqueles que fazem parte do cotidiano do médico generalista.

Podemos delinear três cenários de aprendizagem, e o ideal seria a atuação em todos, quando viável, mas, caso não seja, o ideal é contemplar o máximo possível de cenários.

\section{Internato na atenção básica}

De acordo com a Portaria Nacional de Atenção Básica ${ }^{11}$, a atenção básica se caracteriza por um conjunto de ações de saúde nos âmbitos individual e coletivo que abrange a promoção e proteção da saúde, a prevenção de agravos, o diagnóstico, o tratamento, a reabilitação e a manutenção da saúde. A eficácia dessas ações pode gerar a resolução de $80 \%$ dos problemas de saúde da população.

As DCN de 2014 têm como objetivo claro que o aprendizado médico não seja prioritariamente hospitalar, mas sim centralizado na rede de atenção à saúde, sendo parte fundamental do ensino de prática clínica do futuro médico ${ }^{2,3}$. A existência de um psiquiatra na atenção básica não é uma norma, porém temos desenvolvido prática semanal em uma UBS, na cidade de Bragança Paulista, em que se atendem usuários com transtornos mentais leves, como ansiedade, insônia e depressão leve. A opção por esse tipo de cenário se deu pelo fato de que a grande maioria dos alunos não fará opção pela psiquiatria, e certamente muitos, depois de formados, irão trabalhar em UBS, podendo assim dar resolutividade aos casos mais corriqueiros de saúde mental, sem a necessidade de encaminhamento ao especialista.

Estudo desenvolvido na cidade de Sobral, no Ceará ${ }^{12}$, corrobora tal proposta, pois a maioria dos médicos que atende nas UBSs daquele município relataram que se sentiam despreparados para o atendimento de demandas de saúde mental, identificando deficiências na graduação, em que se priorizava a formação de cunho eminentemente hospitalar, curativo e fora do contexto da atenção comunitária.

No estágio de saúde coletiva, um dos professores de psiquiatria desenvolve, no internato de saúde coletiva, práticas de matriciamento na estratégia de saúde da família.

Internato na rede ambulatorial

Durante o internato de saúde mental, há o desenvolvimento de várias atividades ambulatoriais, como o ambulatório de saúde mental do município de Bragança Paulista, que atende pacientes que por vários motivos não conseguiram se vincular ao Centro de Atenção Psicossocial II (Caps II) municipal.

$\mathrm{Na}$ estrutura do Hospital Universitário São Francisco, existe há 23 anos o Hospital-Dia Escola em Saúde Mental (HD). Remanescente do início da reforma psiquiátrica no Brasil, na década de 1980, e certamente precursor do modelo Caps, o HD apresentava-se como referência para os hospitais psiquiátricos, no intuito de evitar internações prolongadas, bem como para atendimento para pacientes ambulatoriais em crise, a fim de de evitar internação integral. Pelo fato de não haver hospital psiquiátrico em Bragança Paulista, durante muitos anos o HD funcionou como serviço de atendimento a pacientes em crise, por meio de internações parciais (diariamente, no período das 8 às 16 horas) ${ }^{13}$.

Em 2013, foi criada como um anexo ao HD a enfermaria masculina de psiquiatria no Hospital Universitário São Francisco, bem como o Caps II e o Centro de Atenção Psicossocial de Álcool e Outras Drogas (Caps AD) municipais, fato que modificou o perfil de pacientes no HD. Atualmente predominam quadros reativos e transtornos de personalidade.

Além de passarem no HD acompanhando os pacientes internados, os alunos também prestam serviço no ambulatório vinculado ao hospital, referência para os pacientes que receberam alta e são acompanhados ambulatorialmente.

Hoje praticamente inexistem hospitais-dia em nosso país, sendo o Caps a referência de atendimento em saúde mental. Torna-se imperativo que os alunos de graduação vivenciem a experiência no Caps, de modo que possam ter a oportunidade de realizar uma prática de saúde de caráter aberto e comunitário, constituído por equipe multiprofissional que atua sobre a ótica interdisciplinar nos processos de reabilitação psicossocial.

Há também o ambulatório de saúde mental da infância e adolescência, em que o aluno do internato vivencia prática de atendimento na área. Caso no município exista o Caps infantil, tal experiência deve ser vivenciada pelo aluno em uma abrangência mais ampla de cuidados. Como relatado anteriormente, a prática de atendimento de saúde mental da infância e adolescência tanto pode estar vinculada ao internato de saúde mental como à saúde da criança.

A rotina acadêmica ambulatorial envolve o atendimento por parte dos internos e a discussão com o professor para posterior liberação do caso. Depois dos atendimentos, o grupo de internos e o professor fazem uma revisão de todos os casos atendidos no dia, destacando-se os pontos mais significativos para a aprendizagem.

Vale destacar a opção pedagógica de não criar para o internato ambulatórios homogêneos, como ambulatórios de transtorno do humor,

REVISTA BRASILEIRA DE EDUCAÇÃO MÉDICA

4 44 (1) : e005; 2020 
esquizofrenia, fobias etc. A opção é por ambulatórios heterogêneos, pois entendemos a importância do atendimento centrado na pessoa, e não nos sintomas ${ }^{14}$.

Em nossa instituição, o modelo de ambulatório homogêneo está especificado no Programa de Residência Médica de Psiquiatria, cujo propósito é inserir propostas de pesquisa clínica e promover o aprofundamento necessário para a especialização desenvolvida.

Internato hospitalar

$\mathrm{Na}$ formação do internato em saúde mental, certamente o ponto crucial está relacionado à questão hospitalar que remete ainda ao papel do hospital psiquiátrico como cenário de aprendizagem. Entendemos que o hospital geral deve ser o espaço prioritário, se não o único, de práticas na graduação em saúde mental. A experiência do hospital psiquiátrico clássico remete a uma prática psiquiátrica ultrapassada, excludente e cronificante.

Talvez a única razão para que o hospital psiquiátrico seja cenário de aprendizagem consista na existência dele na região da escola médica e na inexistência de enfermaria de psiquiatria em hospital geral.

Desde 2013, a USF possui enfermaria de psiquiatria no Hospital Universitário São Francisco, com 20 leitos masculinos, sendo referência para pacientes psicóticos agudos ou com reagudização, bem como pacientes dependentes químicos. Por possuirmos vínculo contratual com o Centro de Referência de Álcool, Tabaco e Outras Drogas (Cratod) do estado de São Paulo, somos referência em atendimento a pacientes dependentes químicos com comorbidades clínicas. Tal perfil traz benefícios ao interno, pois ele pode vivenciar na enfermaria patologias variadas, como surto psicótico, transtorno bipolar, patologias vinculadas à dependência química (surto psicótico induzido por drogas, abstinência alcoólica, delirium tremens) e comorbidades clínicas associadas (cirrose hepática, diabetes, crise hipertensiva, entre outras).

A rotina da enfermaria é semelhante à de uma enfermaria de qualquer outra especialidade, com visitas diárias, evolução, prescrição, discussão de casos e entrevista com as famílias, mas esse é também um espaço privilegiado para o desenvolvimento de práticas de interconsultoria no hospital geral. A interconsultoria se tornou um importante modelo de integração da psiquiatria com outras áreas da medicina, e, por isso, é fundamental que o aluno participe dessa atividade integrativa para correlacionar a psiquiatria com a medicina geral $^{15}$.

É importante frisar que, em todos os cenários de aprendizagem, deve-se estimular o trabalho interdisciplinar, de modo a levar o estudante de medicina a exercitar o trabalho em equipe. Na USF, vários cursos da área de saúde, como Enfermagem, Psicologia e Fisioterapia, têm, no HD e na enfermaria de psiquiatria, cenários de aprendizagem em saúde mental, o que facilita o trabalho integrativo.

\section{Qual é o número necessário de professores e preceptores?}

No internato médico, a questão da docência é elemento fundamental no processo de ensino. Com o advento das metodologias ativas, o papel do docente foi se modificando na formação médica, já que o aluno passou a ter maior autonomia e capacidade crítica no decorrer do curso. Contudo, no internato, o papel do docente/preceptor é vital nas práticas diárias do internato. Devem-se estimular os atendimentos, as discussões de casos e as reflexões clínicas e psicossociais, o que significa que o docente deve ser uma figura ativa no processo de ensino ${ }^{16}$.
Muitas atividades podem ser feitas em conjunto com os residentes e outros profissionais da equipe, de modo a criar uma dinâmica em que o aluno se sinta parte integrante da equipe, porém não se deve prescindir da atitude docente durante as atividades curriculares.

No internato, todas as atividades necessitam de um docente/ preceptor presente, fato que pode gerar custo alto para a instituição, mas não se pode abrir mão da experiência e do conhecimento por conta das limitações financeiras. Nesse ponto, vale frisar a importância de os professores de disciplinas de Psiquiatria/Saúde Mental participarem dos órgãos colegiados que definem os projetos pedagógicos dos cursos, como comissão de internato, núcleo docente estruturante e coordenação. Enfim, a viabilização de projetos pedagógicos também faz parte de uma política educacional na qual os atores sociais (no caso, os professores) são figuras centrais do processo.

\section{Matriz curricular do internato em saúde mental da USF}

A seguir, apresentamos a matriz curricular do internato médico de saúde mental da USF. Com carga horária de 320 horas, o que corresponde a 40 horas semanais durante oito semanas, dividimos a turma em três grupos que rodiziam quase 14 dias em cada módulo (Anexo 1).

$\mathrm{O}$ grupo A está vinculado ao $\mathrm{HD}$; o $\mathrm{B}$, à enfermaria; e o $\mathrm{C}$, aos ambulatórios. As atividades práticas são separadas, e a turma tem atividades em conjunto nas aulas teóricas e na reunião clínica do módulo. Vale destacar que toda sexta-feira, das 10 às 12 horas, ocorre uma reunião clínica obrigatória de todo o internato (do nono ao $12^{\circ}$ semestre), e, semanalmente, há a apresentação de um caso clínico das diversas especialidades.

Algumas especificidades da matriz serão destacadas, lembrando que a divisão de grupos deve ser feita conforme a necessidade de cada instituição.

No grupo A, enfatizam-se a participação da família e a necessidade de treinamento dos alunos em entrevistar familiares dos pacientes. Tanto na saúde mental como em toda a graduação médica, os alunos têm dificuldade e até desinteresse em entrevistar familiares, pois não entendem que se trata de uma fonte fundamental de informações.

O grupo de cidadania é uma atividade que ocorre sob a supervisão dos residentes, em que os internos preparam algum tema, prioritariamente relacionado à saúde geral, para ser apresentado aos pacientes internados.

A triagem é uma atividade em que pacientes encaminhados ao HD por vários serviços de saúde são avaliados para saber se há ou não indicação de internação parcial.

Quanto ao grupo B, as atividades se desenvolvem como rotina de outras enfermarias gerais. Como esse é o primeiro momento do curso de Medicina em que os alunos vivenciam a experiência de acompanhar pacientes internados, ocorre todo um treinamento para a prática cotidiana em enfermaria.

O grupo $\mathrm{C}$ desenvolve atividades ambulatoriais em cenários distintos, pois entendemos que o perfil dos pacientes se modifica conforme os cenários se apresentam. Vale enfatizar o atendimento na UBS e o ambulatório de psiquiatria infantil que, no nosso caso, está vinculado à saúde mental e não à saúde da criança.

Por fim, vale destacar as atividades teóricas que ocorrem em dois períodos, quando os temas mais prevalentes de psicopatologia, psiquiatria clínica, psiquiatria infantil e psicofarmacologia são discutidos sob a forma de aulas expositivas, discussão teórico-prática ou pelo método tutorial.

A reunião clínica do serviço de psiquiatra ocorre semanalmente, e a escolha do caso clínico se dá pela relevância dele, podendo ser de algum

REVISTA BRASILEIRA DE EDUCAÇ̃̃o MÉDICA

5 44 (1) : e005; 2020 
paciente internado na enfermaria ou no HD. O caso é preparado pelo interno que o acompanha, sob supervisão docente, com a participação de toda equipe técnica na reunião.

\section{CONCLUSÃO}

Após a instituição do Programa Mais Médicos, centenas de escolas médicas foram criadas no Brasil, norteadas pelas DCN de 2014. A experiência do curso de Medicina da USF, no que se refere ao internato médico de saúde mental, estimula a contribuição para a criação de modelos de internato compatíveis com a formação médica esperada. Se no passado o sofrimento psíquico era subestimado, atualmente se tornou uma prioridade na prática médica, portanto é fundamental que os novos médicos tenham em sua formação um olhar atento à saúde mental.

\section{REFERÊNCIAS}

1. Brasil. Lei $\mathrm{n}^{\circ} 12.871 / 2013$, de 22 de outubro de 2013. Institui o Programa Mais Médicos. Diário Oficial da União 23 out 2013.

2. Batista NA, Vilela RQB, Batista SHSS. Educação médica no Brasil. São Paulo: Cortez; 2015

3. Brasil. Diretrizes Curriculares Nacionais do Curso de Medicina. Conselho Nacional de Educação. Câmara de Educação Superior. Resolução no 3, de 20 de junho de 2014.

4. Brasil. Diretrizes Curriculares Nacionais do Curso de Medicina. Conselho Nacional de Educação. Câmara de Educação Superior. Resolução no 4, de 7 de novembro de 2001.

5. Cândido PTS, Batista NA. O internato médico após as Diretrizes Curriculares Nacionais de 2014: um estudo em escolas médicas do estado do Rio de Janeiro. Rev Bras Educ Méd 2019;43(3):36-45.

6. Celeri EHRV, Azevedo RCS, Banzato CEM, Jacintho ACA, Dalgalarrondo P. O ensino de psiquiatria/saúde mental pelo Departamento de Psicologia Médica e Psiquiatria da FCMUnicamp. Cad ABEM 2015;11:48-53.

7. Marcondes E, Mascaretti LA. O internato na graduação médica. In: Gonçalves EL, organizador. Educação médica. São Paulo: Sarvier; 1998. p. 149-66.

8. Zanolli MB, Maciel DT, Streit DS, Muraguchi EMD. Internato médico - Diretrizes Nacionais da ABEM para o internato no curso de Graduação em Medicina, de acordo com as Diretrizes Curriculares Nacionais. In: Lampert J, Bicudo MA, organizadores. 10 anos das Diretrizes Curriculares Nacionais do Curso de Graduação em Medicina. Rio de Janeiro: ABEM, 2014. p. 57-87.
9. Cliquet MB, Rodrigues CIS. Grupo tutorial e a saúde mental no ensino médico. Rev Bras Educ Méd 2016;40(4):591-601.

10. Universidade São Francisco. Projeto Pedagógico do Curso de Medicina. 2018

11. Brasil. Ministério da Saúde. Portaria $\mathrm{n}^{\circ} 2.436$, de 21 de setembro de 2017. Aprova a Política Nacional de Atenção Básica.

12. Pereira AA, Andrade DCL. Estratégia educacional em saúde mental para médicos da atenção básica. Rev Bras Educ Méd. 2018;42(1):4-12.

13. Paulin LF, Teixeira A, Pereira DQ, Villaça D, Santos DM, Poças RCG. Hospital-Dia Escola em Saúde Mental da Universidade São Francisco. Rev Med. USF 1995;13(1):33-9.

14. Paulin LF, Poças RCG. A experiência da Universidade São Francisco com o internato médico de psiquiatria utilizando a metodologia da Aprendizagem Baseada em Problemas. Rev Psiquiatr Rio Gd Sul 2009;31(1):67-72.

15. Botega NJ, organizador. Prática psiquiátrica no hospital geral: interconsulta e emergência. 4. ed. Porto Alegre: Artmed; 2017.

16. Del-Ben CM, Hallak JE, Sponholz Jr A, Marques JM, Labate CM, Contel JO et al. Accuracy of psychiatric diagnosis performed under indirect supervision. Braz J Psychiatry 2005;27(1): 58-62.

\section{CONTRIBUIÇÃO DOS AUTORES}

Os autores tiveram participação efetiva na elaboração do artigo aqui apresentado.

\section{CONFLITO DE INTERESSES}

Os autores declaram não haver conflitos de interesse neste estudo.

\section{ENDEREÇO PARA CORRESPONDÊNCIA}

Luiz Fernando Paulin

Rua Francisca Domingues Camargo de Oliveira 420 - Recanto Amapola - Bragança Paulista

CEP; 12 919-570

luiz.paulin@usf.edu.br 


\section{ANEXO 1}

\begin{tabular}{|c|c|c|c|c|c|}
\hline \multicolumn{6}{|c|}{ Grupo A - Hospital-dia } \\
\hline HORÁRIOS & 2a FEIRA & 3a FEIRA & $4^{\text {a }}$ FEIRA & 5 ${ }^{\text {a }}$ FEIRA & $6^{a}$ FEIRA \\
\hline Das 8 às 10 horas & Grupo de família & Grupo de cidadania & Visita médica & Triagem & $\begin{array}{l}\text { Reunião clínica } \\
\text { Psiquiatria }\end{array}$ \\
\hline Das 10 às 12 horas & Visita médica & Discussão de casos & Visita médica & Evolução & $\begin{array}{l}\text { Reunião geral } \\
\text { Caso clínico }\end{array}$ \\
\hline Das 13 às 17 horas & $\begin{array}{l}\text { Atividade teórica: temas } \\
\text { sobre saúde mental }\end{array}$ & Evolução clínica & $\begin{array}{l}\text { Atividade teórica: temas } \\
\text { sobre saúde mental }\end{array}$ & Entrevista com família & Evolução \\
\hline Das 13 às 17 horas & $\begin{array}{l}\text { Atividade teórica: temas } \\
\text { sobre saúde mental }\end{array}$ & Evolução clínica & $\begin{array}{l}\text { Atividade teórica: temas } \\
\text { sobre saúde mental }\end{array}$ & Triagem & Evolução \\
\hline \multicolumn{6}{|c|}{ Grupo B - Enfermaria } \\
\hline HORÁRIOS & 2a FEIRA & 3a FEIRA & $4^{\text {a }}$ FEIRA & $5^{\text {a }}$ FEIRA & 6aFEIRA \\
\hline Das 8 às 10 horas & Enfermaria de psiquiatria & Enfermaria de psiquiatria & Enfermaria de psiquiatria & Enfermaria de psiquiatria & Reunião clínica \\
\hline Das 10 às 12 horas & Enfermaria de psiquiatria & Grupo de Cidadania & Enfermaria de psiquiatria & Enfermaria de psiquiatria & $\begin{array}{c}\text { Reunião geral Caso } \\
\text { clínico }\end{array}$ \\
\hline Das 13 às 15 horas & $\begin{array}{l}\text { Atividade teórica: temas } \\
\text { sobre saúde mental }\end{array}$ & $\begin{array}{l}\text { Enfermaria de psiquiatria } \\
\text { Reunião teórico-prática }\end{array}$ & $\begin{array}{l}\text { Atividade teórica: temas } \\
\text { sobre saúde mental }\end{array}$ & Grupo de família & Enfermaria de psiquiatria \\
\hline Das 15 às 17 horas & $\begin{array}{l}\text { Atividade teórica: temas } \\
\text { sobre saúde mental }\end{array}$ & $\begin{array}{l}\text { Enfermaria de psiquiatria } \\
\text { Reunião teórico-prática }\end{array}$ & $\begin{array}{l}\text { Atividade teórica: temas } \\
\text { sobre saúde mental }\end{array}$ & Grupo de família & Enfermaria de psiquiatria \\
\hline \multicolumn{6}{|c|}{ Grupo C - Ambulatórios } \\
\hline HORÁRIOS & $2^{\text {a FEIRA }}$ & 3a FEIRA & $4^{\mathrm{a}}$ FEIRA & 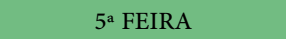 & $6^{\mathrm{a}}$ FEIRA \\
\hline Das 8 às 10 horas & Ambulatório municipal & $\begin{array}{c}\text { Ambulatório do Hospital } \\
\text { Universitário de São Francisco } \\
\text { (Husf) }\end{array}$ & UBS & $\begin{array}{l}\text { Ambulatório } \\
\text { infantil }\end{array}$ & Reunião clínica \\
\hline Das 10 às 12 horas & Ambulatório municipal & Ambulatório do Husf & UBS & $\begin{array}{l}\text { Ambulatório } \\
\text { infantil }\end{array}$ & $\begin{array}{l}\text { Reunião geral } \\
\text { Caso clínico }\end{array}$ \\
\hline Das 13 às 15 horas & $\begin{array}{l}\text { Atividade teórica: temas } \\
\text { sobre saúde mental }\end{array}$ & Ambulatório do Husf & $\begin{array}{l}\text { Atividade teórica: temas } \\
\text { sobre saúde mental }\end{array}$ & $\begin{array}{l}\text { Ambulatório } \\
\text { infantil }\end{array}$ & Ambulatório do Husf \\
\hline Das 15 às 17 horas & $\begin{array}{l}\text { Atividade teórica: temas } \\
\text { sobre saúde mental }\end{array}$ & Ambulatório do Husf & $\begin{array}{l}\text { Atividade teórica: temas } \\
\text { sobre saúde mental }\end{array}$ & $\begin{array}{l}\text { Ambulatório } \\
\text { infantil }\end{array}$ & Ambulatório do Husf \\
\hline
\end{tabular}

